\title{
THEORETICAL CONSIDERATIONS OF THE TL RESPONSE AFTER IRRADIATION-STORAGE PERIODS \\ AT VARIOUS TEMPERATURES
}

\author{
Claudio Furetta \\ CERN, Geneva
}

\begin{abstract}
Theoretical calculations for estimating both the temperature and fading effects on the TL response of dosemeters used in environmental radiation dosimetry are described.

Making use of the first and second order kinetics a set of equations is derived when the TL response is affected both by fading only and by fading in conjunction with environmental background. Then the responses obtained are calculated as a function of the storage temperatures during monitoring time.
\end{abstract}




\section{INTRODUCTION}

Knowledge about the loss of stored signals in thermoluminescent phosphors, commonly called fading, is an important but often neglected factor in environmental radiation monitoring using TLDs, although it is one of the major drawbacks of thermoluminescence dosimetry.

The fading effect has been thoroughly studied for many different types of thermoluminescence materials (Fowler et al., 1965; Suntharalingam and Cameron, 1966; Glenn et al., 1967; Gorbics et al., 1967; Webb, 1967a,b; Orton and Busemann, 1968; Suntharalingam et al., 1968; Nakajima and Hasizume, 1969; Nishita and Hamilton, 1971; Wintle et al., 1971; Sukis, 1971; Becker, 1973, 1974; Gwiazdowski et al., 1974; Johnson, 1974; Mason et al., 1974; Burkhardt et al., 1976; Gross et al., 1976; Furetta and Gennai, 1980, 1981; Nambi, 1982; Tsuda and Mori, 1984).

The usual method used to study the fading is to irradiate the phosphors, at a given temperature, and then read out the samples at different time intervals after storage in constant temperature conditions. In this case it is possible to determine the isothermal decay time constant, i.e. the mean trap life time of the dosimeter peak, and then the related fading rate.

The knowledge of the fading rate becomes very important when TLDs are used in environmental radiation dosimetry.

Another problem arises in this case because the fading factor is temperature dependent and the temperature cannot be considered as a constant during the environmental monitoring time.

The combined effects of background irradiation leading to a progressive storage of a certain charge population into their traps, of fading due to the traps emptying with subsequent radioactive recombination, and of temperature on the fading rate itself have been less thoroughly investigated. 
This problem has been treated only in a few papers (de planque Burke, 1974; Gesell et al., 1980), making use of the Randall and wilkins theory of first order kinetics (1945), and experiments (de Planque Burke, 1974) were performed to examine the adequacy of the mathematical model for describing the above mentioned effects. The results obtained in these experiments differed significantly from the prediction of the theory.

This unsatisfactory agreement between theoretical and experimental data may depend on the inadequate model offered by the Randall and Wilkins theory since many of the most frequently used phosphors do not behave according to the first order kinetics.

In order to take into acount a more general situation, this paper will illustrate the effect of the irradiation temperature on the fading rate, developing mathematical equations obtained considering the model of the first order kinetics as well as the one proposed by Garlick and Gibson (1948) known as the second order kinetics.

\section{MATHEMATICAL MODEL}

\subsection{TL signals when fading occurs}

Let us start with the case when fading occurs during a storage period following the irradiation.

The mathematical treatment is based on the differential equations

$$
\frac{\mathrm{dn}}{\mathrm{dt}}=-\operatorname{sn} \exp \left(-\frac{E}{\mathrm{kT}}\right)
$$

for the first order kinetics, and

$$
\frac{d n}{d t}=-s^{\prime} n^{2} \exp \left(-\frac{E}{k T}\right)
$$

for the second order kinetics. 
The symbols used in the above equations mean:

$\mathrm{dn} / \mathrm{dt}$ is the rate of release of electrons from the traps,

$\mathrm{n}$ is the number of trapped electrons after irradiation,

$E$ is the trap depth,

$\mathrm{k}$ is the Boltzmann's constant,

$s$ and $s^{\prime}\left(=n_{0} s\right)$ are the frequency and pre-experimental factors, respectively,

$T$ is the constant temperature during storage.

The integration of equations (1) and (2) yields

$$
\begin{aligned}
& n=n_{0} \exp [-s t \exp (-E / k T)] \quad \text { 1st order } \\
& n=n_{0}\left[1+n_{0} s t \exp (-E / k T)\right]^{-1} \quad \text { 2nd order }
\end{aligned}
$$

where $n_{0}$ is the initial concentration of the trapped carriers and $s \exp (-E / k T)=\lambda$ is the escape probability and the fading factor.

Since the total amount of light emitted by the phosphor is used in the calculations, $a \phi(t)$ function is introduced to match the $n$ values.

Then, equations (3) and (4) can be written as

$$
\begin{gathered}
\phi(t)=\phi_{0} \exp (-\lambda t) \\
\phi(t)=\phi_{0}\left(1+\phi_{0} \lambda t\right)^{-1}
\end{gathered}
$$

where $\phi_{0}$ is the total TL light if there is no fading. The mean life time $\tau$ of the trapped electrons in their trap is given by the following equations:

$$
\begin{array}{cc}
\tau=s^{-1} \exp (E / k T) & \text { 1st order } \\
\tau^{*}=\left(\phi_{0} s\right)^{-1} \exp (E / k T) & \text { 2nd order }
\end{array}
$$

From Eq. (6) it can be seen how a dependence of the initial value $\phi_{0}$ is introduced. 
Fig. 1 shows the relation between $\lambda$ and the mean life times.

Fig. 1

In Fig. 1, line No. 1, referring to a first order kinetics, illustrates that when the escape probability decreases, the trap life time gets longer.

The line family from No. 2 to No. 10, referring to a second order kinetics, shows the expected dependence of the initial dose. The initial dose increases from line No. 2 to No. 10, while for a given $\lambda$ value $\tau$ increases with decreasing dose.

\subsection{TL signal due to a continuous environmental \\ irradiation in competition with fading}

Whereas the environmental contribution leads to a signal increase, the fading is equivalent to a progressive extinction of the stored information, tending to an asymptotic limit.

In this condition the TL signal is obtained from the following equations:

$$
\begin{array}{ll}
\frac{d n}{d t}=\alpha \dot{B}-s n \exp \left(-\frac{E}{k T}\right) & \text { 1st order } \\
\frac{d n}{d t}=\alpha \dot{B}-s^{\prime} n^{2} \exp \left(-\frac{E}{k T}\right) & \text { 2nd order }
\end{array}
$$

where $\alpha$ is a constant, typical for each TL material, giving the dosemeter sensitivity and $\dot{B}$ is the environmental exposure rate.

The integrals of eqs. (7) and (8) yield the expressions

$$
\begin{aligned}
& \phi(t)=\frac{\phi_{0}}{t \lambda}[1-\exp (-\lambda t)] \quad \text { 1st order } \\
& \phi(t)=\left[\frac{\phi_{0}}{\lambda t}\right]^{1 / 2} \frac{1-\exp \left[-2\left(\phi_{0} \lambda t\right)^{1 / 2}\right]}{1+\exp \left[-2\left(\phi_{0} \lambda t\right)^{1 / 2}\right]} \quad \text { 2nd order }
\end{aligned}
$$

where the substitution $\dot{B} \alpha=\phi_{0} / t$ has been made. 
Figure 2 shows the trend of the signal growth due to the background dose in competition with the inherent fading effect. Eqs. (9) and (10) above have been used for the plots. The asymptotic value for the second order kinetics is reached faster than for the first order kinetics according to the trend of the plots in Fig. 1.

Fig. 2

\section{DISCUSSION}

Making use of eqs. $\left(3^{\prime}\right),\left(4^{\prime}\right),(9)$ and $(10)$, the $\phi(t)$ functions have been calculated at different temperatures from $273^{\circ} \mathrm{K}$ to $323^{\circ} \mathrm{K}$ in steps of 5 degrees and for values of $E=1.6 \mathrm{eV}$ and $\mathrm{s}=10^{19} \mathrm{~s}^{-1}$. Figure 3 shows the plots obtained for a 30-day irradiation-storage period in both cases when fading only occurs (lines 2 and 4 ) and when the environmental irradiation is in competition with fading (lines 1 and 3 ). Lines 1 and 2 correspond to a first order kinetics, while lines 3 and 4 correspond to a second one.

Fig. 3

From the data in this figure it can be seen that the effect of the temperature on the TL response is much larger for a second order peak than for a first order one. If one looks at for example a temperature of $30^{\circ} \mathrm{C}$, the TL response of a phosphor following the second order kinetics (line 3), after a 30-day irradiation-storage period, is about $57 \%$ less than the response from a phosphor following a first order kinetics (line 1) in the same conditions of storage, irradiation and temperature.

From Fig. 3 it is also possible to see that the effect of increasing temperature on the TL response after a 30-day storage period is larger in the second order kinetics than in the first order one. In fact the effect of the temperature is not evident between a temperature range from $273^{\circ} \mathrm{K}$ to $298^{\circ} \mathrm{K}$ for a first order kinetics. After that the TL response decreases by $4 \%$ to $63 \%$ of the non-affected response in the temperature range from $303^{\circ} \mathrm{K}$ to $323^{\circ} \mathrm{K}$ for the first order kinetics. 
The reduction of the TL response is much more evident and faster for a second order kinetics.

This means that one has to pay attention to the kinetics followed by the phosphors used in environmental monitoring experiments.

A very simple method has been recently proposed by Moharil (1984) for determining the order of kinetics. According to this method it is possible to calculate roughly the response expected for dosimeters irradiated and stored at a given temperature.

Alternatively, it is possible to determine experimentally the fading factor using a controlled temperature chamber to simulate the environmental conditions during irradiation.

Another problem arises when the temperature cannot be considered as constant during the monitoring time. This situation is illustrated in Fig. 4, where the average temperatures for each month are plotted in function of the monitoring time of over one year. The monthly mean temperatures, ambient air temperatures, have been obtained from the Service de Climatologie de la suisse romande, Centre météorologique, Genève Aéroport.

Fig. 4

It should be noted that in reality the mean air temperatures reported in the plot are certainly lower than that inside the environmental dosimeter packages exposed to the direct sunlight. For example, Gesell et al. (1980) reported a very large internal temperature growth: a black light-absorbing package produces an increase of $22^{\circ} \mathrm{C}$ above the ambient air temperature. In these conditions it is evident that the loss of information is considerable. 
In practice the new equations to be used are:

$$
\begin{aligned}
& \phi=\phi_{0} \varepsilon_{i} \frac{1}{\lambda_{i} t_{i}}\left[1-\exp \left(-\lambda_{i} t_{i}\right)\right] \quad \text { 1st order } \\
& \phi=\varepsilon_{i}\left[\frac{\phi_{o i}}{\lambda_{i} t_{i}}\right]^{1 / 2} \frac{1-\exp \left[-2\left(\phi_{O i} t_{i} \lambda_{i}\right)^{1 / 2}\right]}{1+\exp \left[-2\left(\phi_{O i} t_{i} \lambda_{i}\right)^{1 / 2}\right]} \text { 2nd order }
\end{aligned}
$$

where $\lambda_{i}$ are the fading factors related to the single monitoring times $t_{i}$ where the temperature could be considered as a constant.

Referring to the results of Gesell et al. (1980) and to Fig. 4, one could suppose that the maximum of the loss of stored information is achieved during the period from May to October, because the increase of temperature inside the dosimeter package should be much higher than during the remaining months of the year. So one could suppose that the fading in January through April and in November-December is negligible. On the other hand, considering the central period (May-October) in Fig. 4 and using Eqs. (11) and (12), it is possible to simulate the loss of stored information. Fig. 5 shows the results given as a percentage of residual TL light as a function of one year monitoring time.

Fig. 5

As it appears from Fig. 5, from a theoretical point of view, the loss of information owing to the effect of high temperature inside the package is drastic for the second order kinetics.

This means, as previously reported (Gesell et al., 1980), that the dosimeter package should have a highly reflecting external surface and the temperature should be continuously monitored during the monitoring time.

\section{CONCLUSION}

The plots in Fig. 3 prove how the effect of temperature can be 
considerable during the monitoring time in environmental radiation dosimetry.

Although the cases studied here are only idealized ones, they can be very useful in understanding what kind of correction factors have to be applied for practical purposes. From the theoretical models it appears also evident that a considerable effort is needed to assess a TLD system for environmental radiation dosimetry.

ACKNOWLEDGEMENTS

The author is indebted to Dr. J.W.N. Tuyn for his many helpful suggestions and discussions. 


\section{REFERENCES}

Becker K., (1973) Long-Term Stability of Film, TLD, and other Integrating Dosimeters in Warm and Humid Climates, ORNL-TM-4297, Oak Ridge National Laboratory, Tennessee.

Becker K., (1974) Stability of $\phi i l m$ and Thermoluminescence Dosimeters in Warm and Humid Climates, Atomkernenergie, 23, 267-274.

Burgkhardt B., Herrera R. and Piesch E., (1976) Fading Characteristics of Different Thermoluminescent Dosimeters, Nucl. Instrum. and Meth., 137, 41-47.

de Planque Burke G., (1974) Thermoluminescence of $\mathrm{CaF}_{2}$ :Mn Exposed at Elevated Temperatures to a constant Rate of Low-Level Radiation, Fourth International Conference on Luminescence Dosimetry, Krakow, Poland, 315-345.

Fowler J.F., Shuttleworth E., Svarcer V., white J.T. and Karzmark C.J., (1965) Fading in Thermoluminescent Lithium Fluoride Used for Radiation Dosimetry, Nature, 207, 997-998.

Furetta C. and Gennai P., (1980) Further Study on the Stability of TLD-900 at Low Radiation Doses, Rad. Eff. Lett., 57, 55-58.

Furetta C. and Gennai P. (1981) An Extensive Study on the Dosimetric Properties of CaSo :Dy Ribbon, TLD-900, in Low Dose Region, Health Phys. $41,674-677$.

Garlick G.F.J. and Gibson A.F. (1948) The Electron Trap Mechanism of Luminescence in Sulphide and Silicate Phosphores, Proc. Phys. Soc. 60, $574-590$.

Gesell T.F., Christian D.C., Gammage R.E. and de Planque G. (1980) Effects of Packaging on the Temperature of Environmental Radiation Dosimeters, Health Phys. 38, 690-696.

Gorbics S.G., Attix F.H. and Pfaff J.H. (1967) Temperature Stability of $\mathrm{CaF}_{2}$ :Mn and LiF(TLD-100) Thermoluminescent Dosimeters, Int. J. Appl. Rad. and Isotope 18, 625-630.

Gross K.C., MC Namara E.J. and Brinck W.L. (1976) Factors Affecting the Use of $\mathrm{CaF}_{\mathrm{S}}: \mathrm{Mn}$ Thermoluminescent Dosimeters for Low-Level Environmental Radiation Monitoring, USEPA Report 600/4-76-035.

Gwiazdowski B., Koczynski H., Kosicka M. and Wolska-Witer M. (1974) The Comparison of Long-Term Parameter stability of Various RTL Detectors Used for Measurements of Natural Background Radiation, Fourth International Conference on Luminescence Dosimetry, Krakow, Poland, 989-1001.

Johnson T.L. (1974) Quantitative Analysis of the Growth and Decay of the TL Peak in LiF (TLD-700), Fourth International Conference on Luminescence Dosimetry, Krakow, Poland, 197-217.

Mason E.W., McKinley A.F., Clark I. and Saunders D. (1974) The Effect of Temperature and Humidity on Lithium Fluoride and Lithium. Borate 
Thermoluminescence Dosimeters, Fourth International Conference on Luminescence Dosimetry, Krakow, Poland, 219-235.

Moharil S.V. (1984) A Simple Method for Obtaining order of Kinetics from Isothermal Decay of Thermoluminescence, J. Phys. D: Appl. Phys. $17,2301-2303$.

Nakajima T. and Hasizume T. (1969) On Applicability of TL Fading to Estimation of Time After Irradiation, Health Phys. 16, 782-783.

Nambi K.S.V. (1982) Self-Dose and Fading of Caso (Dy/TM) and CaF (natural) TL Phosphors in Environmental Radiation Monitoring? Nucl. Instr. and Meth. 197, 453-457.

Nishita H. and Hamilton M. (1971) Influence of Temperature during the Gamma Irradiation Period on the Subsequent Thermoluminescence of Soils and $\mathrm{LiF}$ and $\mathrm{CaF}_{2}$ Dosimeters, Soil Science, 111, 393-398.

Orton C.G. and Busemann E. (1968) Initial Fading Characteristics of LiF-Teflon Micronods, Second International Conference on Luminescence Dosimetry, Gatlinburg, 632-638.

Randall J.T. and Wilkins M.E.F. (1945) Phosphorescence and Electron Traps, Proc. R. Soc., 184A, 366-407.

Sukis D.R. (1971) Thermoluminescent Properties of $\mathrm{CaF}_{2}$ :Dy TLD's, IEEE Trans. Nucl. Sci., NS-18, 185-189.

Suntharlingam N. and Cameron J.R., Shuttleworth E., West M. and Fowler J.R. (1968) Fading Characteristics of Thermoluminescent Lithium Fluoride, Phys. Med. Biol. 13, 97-104.

Tsuda M. and Mori T. (1984) The Short-Term Fading of TLDs, Nucl. Instr. and Meth. in Phys. Res. 219, 588-591.

Webb G.A.M. (1967a) Fading in Thermoluminescent Lithium Fluoride after Irradiation with Low Doses, Brit. J. Appl. Phys. 18, 7-11.

Webb G.A.M. (1967b) Fading in Thermoluminescent Lithium Fluoride after Irradiation with Gamma-Ray Doses up to 1000 Rad, Brit. J. Appl. Phys. 18, 1567-1571.

Winter A.G., Aitken M.J. and Huxtable J. (1971) Abnormal Thermoluminescence Fading Characteristics, Third International Conference on Luminescence Dosimetry, Risö, Risö Report No. 249, $105-131$. 


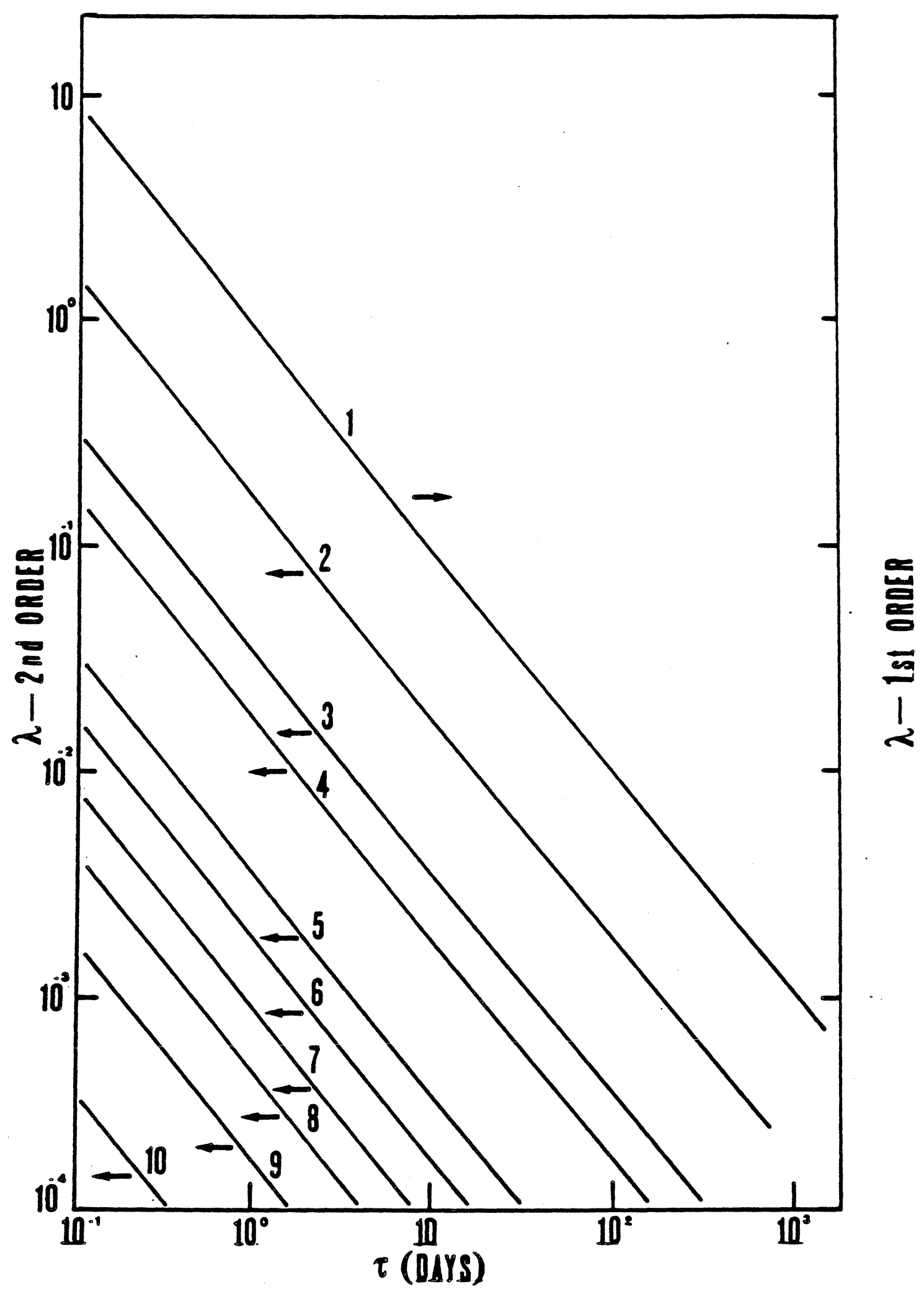

Fig. $q$ Relation between $Y$ and $\tau$ for both 1 st and 2nd order kinetics. The lines have been obtained making use of Eqs. (5) and (6). For the second order kinetics the dependence on initial dose is put into evidence by the line shift. Fron lines No. 2 to No. $10,=10,50,100,500,1000,2000,4000,10000$ and 50000 . 


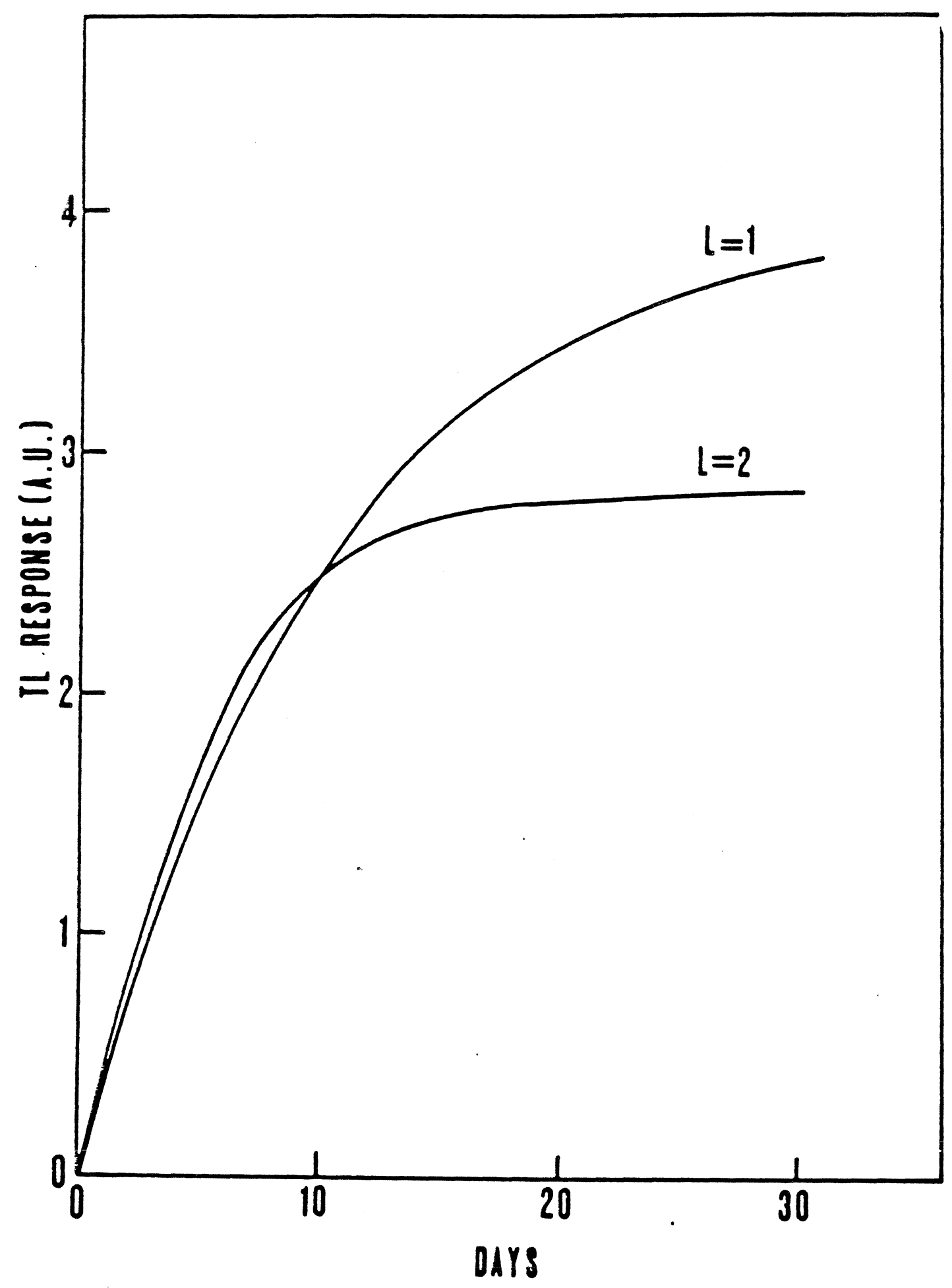

Fig. 2 Trend of the background dose in competition with fading. Eqs. $(9)$ and $(10)$ have been used for ist $(L=1)$ and 2 nd $(L=2)$ order, respectively, with $\tau=10$ days. 


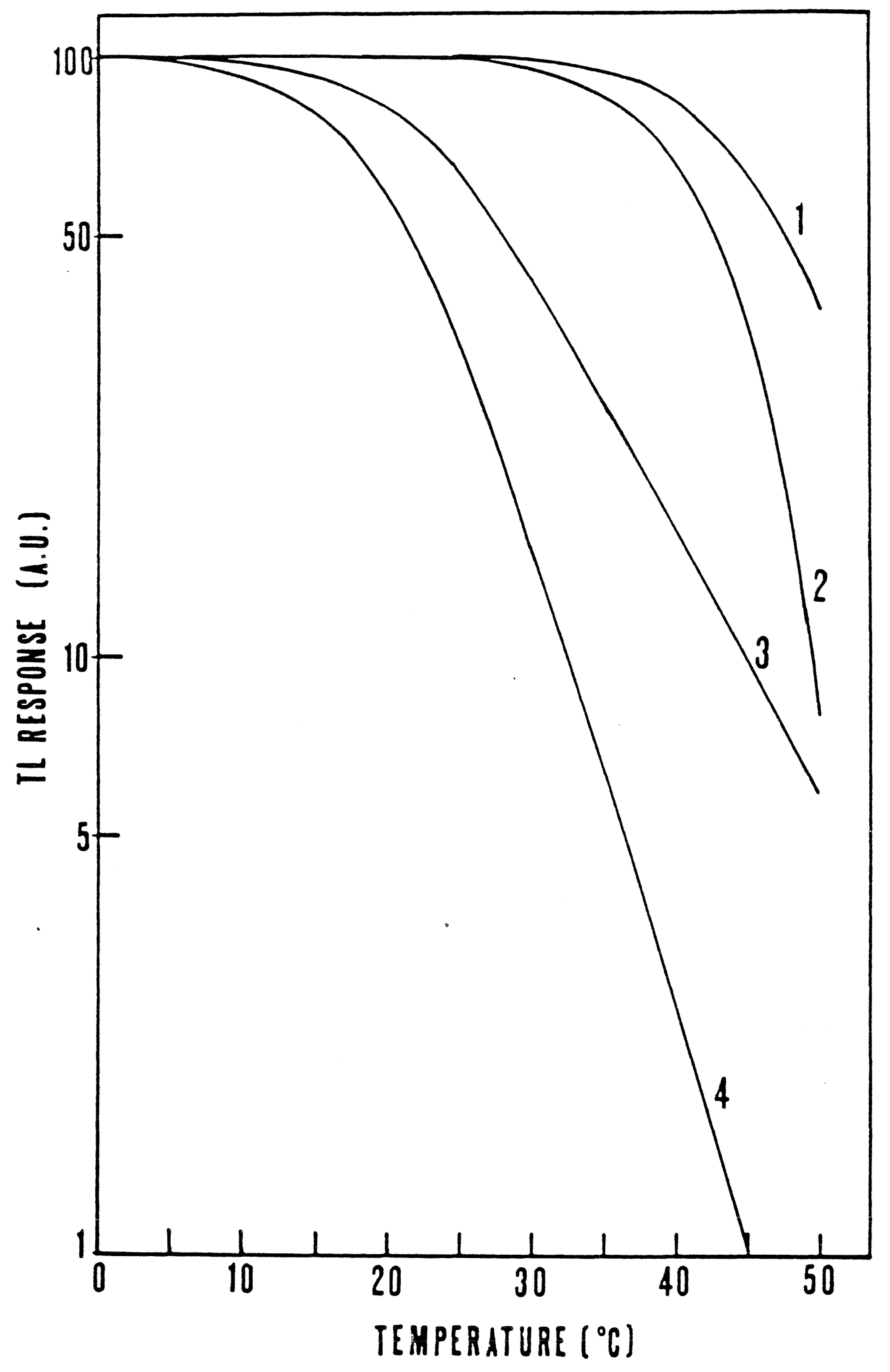

Fig. 3 Calculated response for a continuous 30-day background irradiation in competition with fading as a function of temperature (lines 1 and 3 ) and for an initial irradiation (lines 2 and 4 ). Lines 1 and 2 correspond to a 1 st order kinetics, lines 3 and 4 to a 2 nd one. 


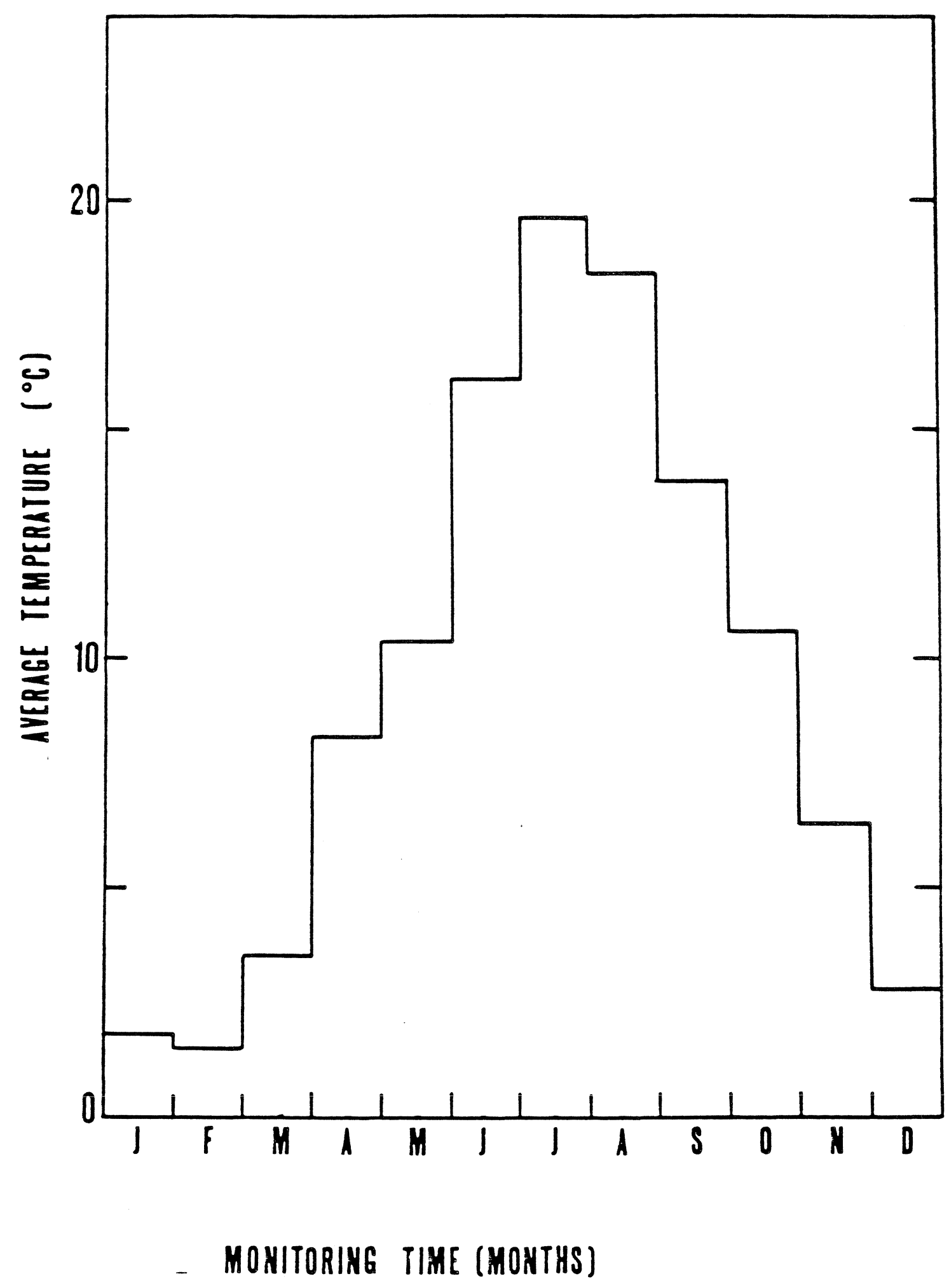

Fig. 4 Monthly average temperatures during 1984 in the airport area of Geneva. 


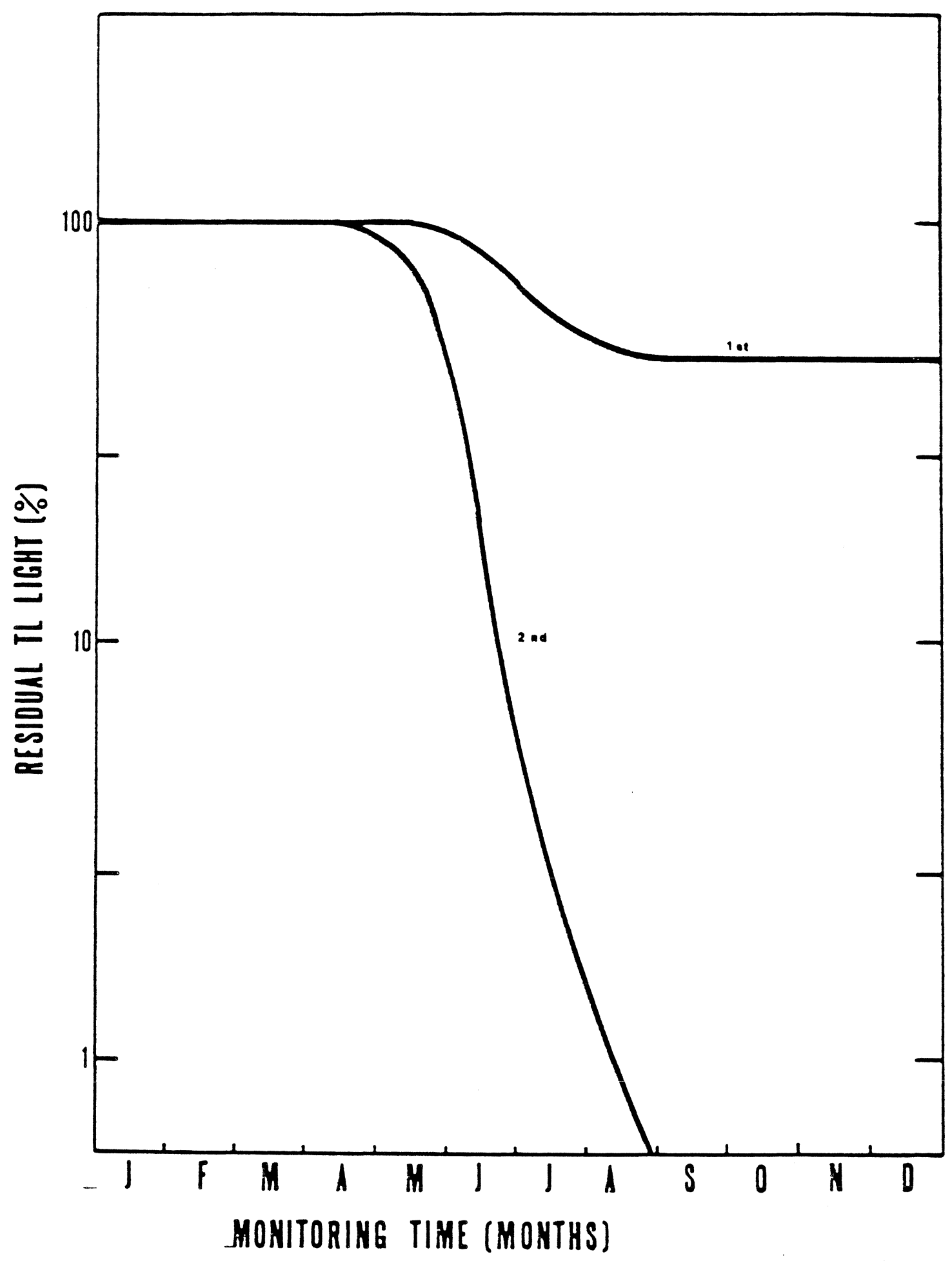

Fig. 5 Residual IL light, as a percentage, in function of the monitoring time. 